\section{() OPEN ACCESS}

\title{
The earlier, the better or the worse? Towards accurate management of patients with arthralgia at risk for RA
}

\author{
Annette van der Helm-van Mil @ (1,2 Robert B M Landewé3,4
}

\begin{abstract}
Handling editor Josef $S$ Smolen

${ }^{1}$ Rheumatology, Leiden University Medical Center, Leiden, The Netherlands

${ }^{2}$ Rheumatology, Erasmus Medical Center, Rotterdam, The Netherlands

${ }^{3}$ Amsterdam Rheumatology Center, AMC, Amsterdam, The Netherlands

${ }^{4}$ Rheumatology, Zuyderland MC, Heerlen, The Netherlands
\end{abstract}

\section{Correspondence to}

Professor Annette van der Helmvan Mil, Rheumatology, Leiden University Medical Center, Leiden, Netherlands: A.H.M.van_der_Helm@lumc.nl

Received 26 November 2019 Accepted 2 January 2020 Published Online First

8 January 2020

\section{Check for updates}

(C) Author(s) (or their employer(s)) 2020. Re-use permitted under CC BY-NC. No commercial re-use. See rights and permissions. Published by BMJ.

To cite: van der Helmvan Mil A, Landewé RBM. Ann Rheum Dis

2020;79:312-315.

\section{ABSTRACT}

The favourable long-term results of early treatment in patients with classified rheumatoid arthritis have resulted in an increasing interest in the diseases phases preceding clinical arthritis. The hypothesis to test is that an intervention in these early phases may better prevent or reduce disease persistence than an intervention when arthritis has become clinically manifest. While several placebo-controlled trials are still ongoing, to date there is no firm evidence that this hypothesis truly holds.

Therefore, it is important to reflect on the current status of arthralgia preceding clinical arthritis. Inherent to every new field of research, attitudes are conflicting, with opinions propagating innovation (based on the fear of undertreatment) on the one hand, and critical sounds pleading for more restraint (fear of overtreatment) on the other hand. In this Viewpoint, we will examine these divergent opinions, relate them to a preferred ultimate scenario and provide considerations for future studies and daily practice.

\section{INTRODUCTION}

Early treatment start has become the cornerstone of the management of early arthritis and rheumatoid arthritis (RA). ${ }^{12}$ This concept has resulted in an increasing interest in the period before swollen joints actually appear, with the underlying assumption that disease processes that are still developing are more susceptible to permanent modification. Treatment success in the prearthritis phase will for instance be reflected by a lower incidence of persistent clinical arthritis, as disease persistence can hardly be affected anymore when treatment is initiated only when arthritis has appeared. Whether disease modifying anti-rheumatic drug (DMARD) treatment started in the prearthritis phase is truly more effective will become clear from the currently ongoing placebo-controlled proof-of-concept trials. Results will become available in the next 2 years. ${ }^{3-6}$

Therefore, it is important now to reflect on the status of the field of arthralgia preceding clinical arthritis. How should we deal with the temptations of scientific progress? Should we already try to positively influence the lives of current individuals presenting with arthralgia, but still without arthritis? How will such a practice influence the chance that we will ever get real evidence-based treatments in this field that are beyond beliefs? In this Viewpoint, we will investigate these questions. We will search for a trade-off between opinions propagating innovation on the one hand and critical sounds pleading for restraint on the other hand, conflicting opinions that are inherent to new fields of research.

\section{THE DILEMMA}

In the absence of evidence for the value of drug interventions in the phase preceding clinical arthritis, several scenarios may apply to describe the current thinking.

The first is the scenario of hope. To cite the American philosopher J. Dewey 'every great advance in science has issued from a new audacity of imagination'. Treatment of RA has considerably improved and the hope is to further improve the lives of patients with RA, curing the disease or even prevent it. This hope is fuelled by promising results of DMARD-intervention in early RA. The contribution of timing of treatment to treatment success has driven researchers to study biological mechanisms that precede clinical arthritis. In clinical practice, patients with arthralgia suspicious of progression to RA, but yet without clinically apparent arthritis, are increasingly recognised. Until recently, these patients were sent home with the advice to come back in case of clinical arthritis. To date, some clinicians feel the need to start DMARD-therapy prior to the development of clinical arthritis, especially if laboratory or imaging findings suggest that RA is looming. They hope to add value to the lives of these patients and argue that-when ongoing trials will teach us that treatment in the prearthritis phase is effective-withholding DMARD-treatment would in hindsight mean undertreatment, a situation that they would rather avoid.

The second scenario is that of criticism or perhaps pessimism. The underlying sentiment is that, now disease activity can be so well suppressed in most RA patients, too early treatment may do more harm than good. In other words, very early treatment start could result in 'overdiagnosis' and overtreatment. In this view, the valuable progress that has been achieved has created a new problem. The British psychologist Havelock Ellis described it as follows: 'What we call progress is the exchange of one nuisance for another nuisance'.

As so often, a third scenario that appropriately balances the risks of undertreatment and overtreatment is likely the ideal scenario.

\section{LOOKING BACK}

Balancing the risks of undertreatment and overtreatment in RA is not new. Fifteen years ago, similar discussions pertained to patients with undifferentiated arthritis (UA), that is, patients with clinically evident arthritis that do not fulfil classification criteria for RA or other inflammatory arthritides. Initially, patients diagnosed with UA were not treated with DMARDs. Only after ample validation of models predicting the risks in individual patients with acceptable accuracy, ${ }^{7-12}$ the 
notion occurred that UA should better be treated. Finally, in order to be able to also classify patients earlier in time, novel classification criteria for RA were developed. ${ }^{13}$ Intriguingly, all placebo-controlled randomised clinical trials in patients with UA were negative for their primary endpoint, the fulfilment of classification criteria for RA. ${ }^{14-18}$ These negative results may be explained by methodological limitations, such as small sample sizes and absence of risk stratification at inclusion. They may also indicate that-if prevention of RA is the ultimate goal-the phase of UA is too late to start DMARD-treatment. Posthoc analysis of a trial with methotrexate in the subgroup of patients with a high risk to progress to RA showed statistically significant and clinically relevant effects. ${ }^{19}$ A meta-analysis of all trials performed in patients with UA provided similar results that were statistically significant. ${ }^{20}$ To what extent overtreatment or undertreatment exists in UA will forever remain unclear, because DMARD treatment of patients with UA is common practice now. In the absence of solid scientific evidence from clinical trials, treatment decisions are guided by clinical expertise and personal experiences.

\section{WHAT CAN WE LEARN FROM THE RECENT PAST?}

In light of the preferred third scenario, important learning points from the past 15 years include the need to conduct well-designed placebo-controlled clinical trials, the need to base our future actions on the results of these trials and to refrain from implementing anticipated results in daily practice that are not (yet) existent.

Adequate trial design means requirements for statistical power, eligible patients, preferred outcome(s) and follow-up duration. Sufficient statistical power seems a trivial requirement but is tricky, because statistical power depends on the difficult to estimate proportion of patients who will get the ultimate outcome (RA). The failure of previous clinical trials with either UA or arthralgia to meet their primary endpoint has been attributed to insufficient sample sizes.

Choosing the best primary outcome is also not straightforward. The primary outcome used so far was fulfilment of RA according to classification criteria. The question is whether this outcome best reflects added value to patients. Nowadays the involvement of patient partners in research has paid off and patients have indicated that current disease burden is mostly caused by pain, fatigue and functional impairments. ${ }^{21}$ From this perspective, added value may better be expressed as the possibility to acquire symptom resolution and maintain a normal daily living (including work). There is also increasing pressure from society to spend healthcare resources more parsimoniously, especially in light of the risen drug expenses for RA. Furthermore, from a methodological perspective, the achievement of an outcome at a single point in time is not reflective of the subsequent disease course. More specifically, the occurrence of clinically apparent arthritis (or RA) at a single time point does not say anything about whether the disease will be selflimiting, whether remission will be rapidly achievable with firstline therapy, whether a DMARD-free status can be achieved over time or whether the disease will be persistently active or poorly responsive. Sustained DMARD-free remission may be a better outcome, as it includes a form of persistence in its definition. ${ }^{22}$ Altogether, a long-term follow-up of patients included in pre-RA trials is required to evaluate if results are sustainable and valuable, which is challenging as trials generally tend to shorten the follow-up duration. Considerations are summarised in box 1 .
Box 1 Considerations on treatment of patients with arthralgia suspicious for progression to RA

- There is no evidence that starting DMARD treatment in this disease phase is effective.

- Several proof-of-concept trials are currently ongoing.

- Subsequent trials require long-term follow-up to determine if outcomes (absence of clinical arthritis, absence of persistent arthritis, achieving DMARD-free status) are sustainable. These trials should include outcomes that reflect real value to patients, such as patient-reported symptoms, functional ability and workability.

- The EULAR definition of arthralgia suspicious for progression to RA confers a high sensitivity for RA development but only a moderate specificity. Adding information from other biomarkers is needed to further increase specificity.

- Currently, there is no validated risk stratification method to reliably estimate the risk of progressing to RA. Analyses on a combination of markers in relation to the natural disease course in all relevant longitudinal data-sets are needed to achieve this.

- In daily clinical practice, rheumatologists may wish to balance the risks of overtreatment and undertreatment in patients with arthralgia suspicious for progression to RA. However, absence of evidence on risk estimations and on efficacy currently favours a decision not to treat arthralgia with DMARDs in the absence of clinical arthritis. Furthermore, evaluation of the natural course will shed light on risks in the nearby future.

RA, rheumatoid arthritis.

\section{IDENTIFYING PERSONS WITH ARTHRALGIA AT RISK FOR RA}

Defining the population of patients with arthralgia but without clinical arthritis, and who are supposedly at risk for RA, is another crucial element. The risk influences the required sample size: at a similar power, a larger sample is required if the risk for RA is low or moderate, as compared with a scenario in which the risk is high. Moreover, overtreatment becomes more of an issue if the actual proportion that will develop RA is smaller. Apart from accurate, the risk estimation should be robust and validated in data from different centres and countries. Since identifying RA purely based on underlying biological markers is still impossible, a proper diagnosis must rely on a combination of features and pattern recognition. A combination of clinical symptoms and signs suggestive of future RA has been developed, resulting in the EULAR definition of arthralgia suspicious for progression to RA. ${ }^{23}$ The clinical definition has shown to be highly sensitive when tested against the external standard 'expert diagnosis of RA' in cross-sectional studies and actual RA development in longitudinal studies. ${ }^{23}{ }^{24}$ In order to obtain a sufficiently high specificity, the clinical definition should be combined with the results of biomarkers. Accumulating evidence suggest that autoantibodies and imaging-detected subclinical inflammation are the most promising biomarkers. A non-systematic look in the literature yields several different prediction models that have been construed. ${ }^{25-29}$ Unfortunately, none of these has reported cross-validation in independent centres, which leaves researchers with residual uncertainty. Collaboration based on data sharing across centres and obtaining consensus on the preferred methodology is needed to optimally define a population at high risk to be included in future trials. 


\section{ASSESSING ACCURACY OF IDENTIFYING RISK FOR RA}

Risk estimators for groups of patients in trials differ principally from those for individuals in clinical practice. In individual patients, positive and negative predictive values, expressing the likelihoods of contracting a disease or remain free of that, are the pivotal estimates. Ideally, these should approach 100\%. This is an almost impossible scenario in rheumatology. Diagnostic criteria that are intended to be used in individual patients are therefore no longer pursued by the professional organisations. Initially, prognostic research has focused on the ability to identify patients at risk for RA: the sensitivity. However, as a high sensitivity harbours the risks of 'overclassification' and overtreatment, attention has shifted from high sensitivity to high specificity (or properly recognising the persons that will not develop RA). The best risk classification model therefore has a high accuracy based on high sensitivity and high specificity. Designing and validating such a model is a 'herculean task' since sensitivity and specificity tend to operate in opposite directions. A factor that further complicates the matter is that even in the presence of a high specificity absolute likelihoods can still be low (Bayesian rule). A good example is the ACPA-test, with a documented specificity of $98 \%$, that in populations with a low prior risk of RA, such as the general population, yields an individual likelihood of RA development of only $5 \%$, corresponding to a likelihood of $95 \%$ of not getting RA. ${ }^{30}{ }^{31}$ In more selected populations with higher prior risk, higher positive predictive values (PPVs) can be found. ${ }^{28} 32$ 'The pre-RA period' is a continuum that extends from health to the time immediately before the development of clinical arthritis and diagnosis or classification of RA. The risk of persistent disease varies by the place in this spectrum; risk stratification algorithms should therefore be developed for subpopulations separately.

\section{CONSIDERATIONS OTHER THAN ACCURACY}

It is arguable, though, whether trials on DMARD-treatment in at risk populations are only justified in the context of optimally accurate prediction models. Whether overtreatment or undertreatment, due to suboptimal accuracy, will be considered socially acceptable depends on many factors such as the likelihood of harm (toxicity of treatment, psychological harm caused by uncertainty about getting ill), treatment expenses, and consequences of missing a diagnosis. Satisfactory answers can only be provided by international consensus about preferable risk stratification models, validation of such models in international databases with data about the natural course and all levels of variability. An estimation of the added value for individuals should be part of discussion. These discussions that involve all stakeholders may ultimately lead to consensus on what is the best trade-off between 'ideal' and 'feasible'. Importantly, persons at risk should be included in these discussions, as their beliefs and preferences will predict treatment uptake. ${ }^{33}$ Optimal participation in this process requires that information is lucid, fair and comprehensible to lay-people. ${ }^{34}$

So far, we have focused on pharmacological interventions in selected populations, but we appreciate the relevance of generic lifestyle interventions such as smoking cessation. Such interventions have a lower risk of harm than DMARDs and are also associated with other positive public health effects.

\section{WHAT DOES THE CURRENT SITUATION IMPLY FOR PATIENTS WITH ARTHRALGIA SUSPICIOUS FOR PROGRESSION TO RA IN DAILY PRACTICE?}

Since there is no broadly accepted method to identify patients at risk for RA with sufficient precision, scenario three in which both undertreatment and overtreatment are minimised does not yet exist. As discussed in the previous paragraphs, long-term observational data on the natural course and outcome are crucial for achieving accurate prognostication. Evaluation of biosamples from longitudinal cohort studies may help elucidating mechanisms that drive the progression from arthralgia to clinically evident RA and may reveal targets for potential intervention. Treating patients before they present with clinical arthritis will make it impossible to obtain reliable information about the natural course of the disease. We may then end up in the belief that we are treating the correct patients, but without appropriate scientific endorsement. This scenario bears resemblance to the current situation for patients with UA. For now, we should learn lessons from the past and remain reluctant to start treatment in the absence of clinical arthritis.

Contributors Both authors have written the manuscript and approved the final version.

Funding The authors have not declared a specific grant for this research from any funding agency in the public, commercial or not-for-profit sectors.

Competing interests None declared.

Patient consent for publication Not required.

Provenance and peer review Not commissioned; externally peer reviewed.

Open access This is an open access article distributed in accordance with the Creative Commons Attribution Non Commercial (CC BY-NC 4.0) license, which permits others to distribute, remix, adapt, build upon this work non-commercially, and license their derivative works on different terms, provided the original work is properly cited, appropriate credit is given, any changes made indicated, and the use is non-commercial. See: http://creativecommons.org/licenses/by-nc/4.0/.

\section{ORCID iD}

Annette van der Helm-van Mil http://orcid.org/0000-0001-8572-1437

\section{REFERENCES}

1 Combe B, Landewe R, Daien Cl, et al. 2016 update of the EULAR recommendations for the management of early arthritis. Ann Rheum Dis 2017;76:948-59.

2 Smolen JS, Landewé R, Bijlsma J, et al. EULAR recommendations for the management of rheumatoid arthritis with synthetic and biological disease-modifying antirheumatic drugs: 2016 update. Ann Rheum Dis 2017;76:960-77.

3 Available: https://www.trialregister.nl/trial/4599

4 EudraCT number: 2013-003413-18

5 EudraCT number: 2014-000555-93

6 Available: https://clinicaltrials.gov/ct2/show/NCT02603146

7 van der Helm-van Mil AHM, le Cessie S, van Dongen $\mathrm{H}$, et al. A prediction rule for disease outcome in patients with recent-onset undifferentiated arthritis: how to guide individual treatment decisions. Arthritis Rheum 2007;56:433-40.

8 van der Helm-van Mil AHM, Detert J, le Cessie S, et al. Validation of a prediction rule for disease outcome in patients with recent-onset undifferentiated arthritis: moving toward individualized treatment decision-making. Arthritis Rheum 2008;58:2241-7.

9 McNally E, Keogh C, Galvin R, et al. Diagnostic accuracy of a clinical prediction rule $(C P R)$ for identifying patients with recent-onset undifferentiated arthritis who are at a high risk of developing rheumatoid arthritis: a systematic review and meta-analysis. Semin Arthritis Rheum 2014:43:498-507.

10 Kuriya B, Cheng CK, Chen HM, et al. Validation of a prediction rule for development of rheumatoid arthritis in patients with early undifferentiated arthritis. Ann Rheum Dis 2009;68:1482-5

11 Ghosh K, Chatterjee A, Ghosh S, et al. Validation of Leiden score in predicting progression of rheumatoid arthritis in undifferentiated arthritis in Indian population. Ann Med Health Sci Res 2016:6:205-10.

12 Arana-Guajardo A, Pérez-Barbosa L, Vega-Morales D, et al. Application of a prediction model for the progression of rheumatoid arthritis in patients with undifferentiated arthritis. Reumatol Clin 2014;10:360-3.

13 Aletaha D, Neogi T, Silman AJ, et al. 2010 rheumatoid arthritis classification criteria: an American College of Rheumatology/European League against rheumatism collaborative initiative. Ann Rheum Dis 2010;69:1580-8.

14 van Dongen $\mathrm{H}$, van Aken J, Lard LR, et al. Efficacy of methotrexate treatment in patients with probable rheumatoid arthritis: a double-blind, randomized, placebocontrolled trial. Arthritis Rheum 2007:56:1424-32.

15 Saleem B, Mackie S, Quinn M, et al. Does the use of tumour necrosis factor antagonist therapy in poor prognosis, undifferentiated arthritis prevent progression to rheumatoid arthritis? Ann Rheum Dis 2008;67:1178-80. 
16 Verstappen SMM, McCoy MJ, Roberts C, et al. Beneficial effects of a 3-week course of intramuscular glucocorticoid injections in patients with very early inflammatory polyarthritis: results of the STIVEA trial. Ann Rheum Dis 2010;69:503-9.

17 Machold KP, Landewé R, Smolen JS, et al. The stop arthritis very early (save) trial, an international multicentre, randomised, double-blind, placebo-controlled trial on glucocorticoids in very early arthritis. Ann Rheum Dis 2010;69:495-502.

18 Emery P, Durez P, Dougados M, et al. Impact of T-cell costimulation modulation in patients with undifferentiated inflammatory arthritis or very early rheumatoid arthritis: a clinical and imaging study of abatacept (the adjust trial). Ann Rheum Dis 2010;69:510-6.

19 Burgers LE, Allaart CF, Huizinga TWJ, et al. Brief report: clinical trials aiming to prevent rheumatoid arthritis cannot detect prevention without adequate risk stratification: a trial of methotrexate versus placebo in undifferentiated arthritis as an example. Arthritis Rheumatol 2017;69:926-31.

20 Hilliquin $\mathrm{S}$, Hugues B, Mitrovic $\mathrm{S}$, et al. Ability of disease-modifying antirheumatic drugs to prevent or delay rheumatoid arthritis onset: a systematic literature review and meta-analysis. Ann Rheum Dis 2018;77:1099-106.

21 van Tuyl LHD, Sadlonova M, Hewlett $\mathrm{S}$, et al. The patient perspective on absence of disease activity in rheumatoid arthritis: a survey to identify key domains of patientperceived remission. Ann Rheum Dis 2017;76:855-61.

22 Ajeganova S, van Steenbergen HW, van Nies JAB, et al. Disease-modifying antirheumatic drug-free sustained remission in rheumatoid arthritis: an increasingly achievable outcome with subsidence of disease symptoms. Ann Rheum Dis 2016;75:867-73.

23 van Steenbergen HW, Aletaha D, Beaart-van de Voorde LJJ, et al. EULAR definition of arthralgia suspicious for progression to rheumatoid arthritis. Ann Rheum Dis 2017;76:491-6.

24 Burgers LE, Siljehult F, Ten Brinck RM, et al. Validation of the EULAR definition of arthralgia suspicious for progression to rheumatoid arthritis. Rheumatology 2017;56:2123-8.
25 Rakieh C, Nam JL, Hunt L, et al. Predicting the development of clinical arthritis in antiCCP positive individuals with non-specific musculoskeletal symptoms: a prospective observational cohort study. Ann Rheum Dis 2015;74:1659-66.

26 Nam JL, Hensor EMA, Hunt L, et al. Ultrasound findings predict progression to inflammatory arthritis in anti-CCP antibody-positive patients without clinical synovitis. Ann Rheum Dis 2016;75:2060-7.

27 van de Stadt $\mathrm{LA}$, Witte $\mathrm{BI}, \mathrm{Bos} \mathrm{WH}$, et al. A prediction rule for the development of arthritis in seropositive arthralgia patients. Ann Rheum Dis 2013;72:1920-6.

28 van Steenbergen HW, Mangnus L, Reijnierse M, et al. Clinical factors, anticitrullinated peptide antibodies and MRI-detected subclinical inflammation in relation to progression from clinically suspect arthralgia to arthritis. Ann Rheum Dis 2016;75:1824-30.

29 Hensvold A, Kisten Y, Circiumaru A, et al. Development of ultrasound detectable arthritis among AcpA positive subjects with musculoskeletal symptoms: the risk RA prospective study. Arthritis Rheumatol 2019;71.

30 Hensvold AH, Frisell T, Magnusson PKE, et al. How well do ACPA discriminate and predict RA in the general population: a study based on 12590 populationrepresentative Swedish twins. Ann Rheum Dis 2017;76:119-25.

31 Haji Y, Rokutanda R, Kishimoto M, et al. Anti-cyclic citrullinated protein antibody (ACPA) positivity in general population and follow-up results for ACPA positive persons [abstract]. Arthritis Rheumatol 2016;68:512.

32 Ramos-Remus C, Castillo-Ortiz JD, Aguilar-Lozano L, et al. Autoantibodies in prediction of the development of rheumatoid arthritis among healthy relatives of patients with the disease. Arthritis Rheumatol 2015;67:2837-44.

33 Falahee M, Finckh A, Raza K, et al. Preferences of patients and at-risk individuals for preventive approaches to rheumatoid arthritis. Clin Ther 2019;41:1346-54.

34 Mosor E, Stoffer-Marx M, Steiner G, et al. I would never take preventive medication! Perspectives and information needs of people who underwent predictive tests for rheumatoid arthritis. Arthritis Care Res 2019. 